\title{
RELATIONSHIPS AMONG TRANSFORMS, CONVOLUTIONS, AND FIRST VARIATIONS
}

\author{
JEONG GYOO KIM, JUNG WON KO, CHULL PARK, and DAVID SKOUG
}

(Received 7 October 1997)

\begin{abstract}
In this paper, we establish several interesting relationships involving the Fourier-Feynman transform, the convolution product, and the first variation for functionals $F$ on Wiener space of the form

$$
F(x)=f\left(\left\langle\alpha_{1}, x\right\rangle, \ldots,\left\langle\alpha_{n}, x\right\rangle\right),
$$

where $\left\langle\alpha_{j}, x\right\rangle$ denotes the Paley-Wiener-Zygmund stochastic integral $\int_{0}^{T} \alpha_{j}(t) d x(t)$.

Keywords and phrases. Fourier-Feynman transform, convolution product, first variation, Feynman integral.
\end{abstract}

1991 Mathematics Subject Classification. 28C20.

1. Introduction. Let $C_{0}[0, T]$ denote one-parameter Wiener space; that is the space of $\mathbb{R}$-valued continuous functions $x$ on $[0, T]$ with $x(0)=0$. The concept of an $L_{1}$ analytic Fourier-Feynman transform was introduced by Brue in [1]. In [3], Cameron and Storvick introduced an $L_{2}$ analytic Fourier-Feynman transform. In [11], Johnson and Skoug developed an $L_{p}$ analytic Fourier-Feynman transform theory for $1 \leq p \leq 2$ which extended the results in [1,3] and gave various relationships between the $L_{1}$ and the $L_{2}$ theories. In [7], Huffman, Park, and Skoug defined a convolution product for functionals on Wiener space and in $[9,7,8]$, they established various results involving transforms and convolutions. In [5], Cameron and Storvick evaluated the Feynman integral of the first variation of certain functionals on Wiener space and in [13], Park, Skoug, and Storvick examined various relationships existing among the first variation, the Fourier-Feynman transform, and the convolution product for functionals on Wiener space which belong to Banach algebra $\mathscr{S}$ in [4].

Section 3 of this paper includes all the relationships involving exactly two of the three concepts of "transform," "convolution product," and "first variation" of functionals of the type mentioned in the abstract. In Section 4, we examine all the relationships involving all three of these concepts, but where each concept is used exactly once.

2. Definitions and preliminaries. Let $\mu$ denote the class of all Wiener measurable subsets of $C_{0}[0, T]$ and let $m$ denote Wiener measure. $\left(C_{0}[0, T], \mu, m\right)$ is a complete measure space and we denote the Wiener integral of a functional $F$ by $\int_{C_{0}[0, T]}$ $F(x) m(d x)$.

A subset $E$ of $C_{0}[0, T]$ is said to be scale-invariant measurable $[6,12]$ provided $\rho E \in$ $\mu$ for all $\rho>0$, and a scale-invariant measurable set $N$ is said to be scale-invariant null 
provided $m(\rho N)=0$ for each $\rho>0$. A property that holds except on a scale-invariant null set is said to hold scale-invariant almost everywhere (s-a.e.). If two functionals $F$ and $G$ are equal s-a.e., we write $F \approx G$.

Let $\mathbb{C}_{+}=\{\lambda \in \mathbb{C}: \operatorname{Re} \lambda>0\}$ and $\mathbb{C}_{+}^{\sim}=\{\lambda \in \mathbb{C}: \lambda \neq 0$ and $\operatorname{Re} \lambda \geq 0\}$. Let $F$ be a $\mathbb{C}$-valued scale-invariant measurable functional on $C_{0}[0, T]$ such that

$$
J(\lambda)=\int_{C_{0}[0, T]} F\left(\lambda^{-1 / 2} x\right) m(d x)
$$

exists for all $\lambda>0$. If there exists a function $J^{*}(\lambda)$ analytic in $\mathbb{C}_{+}$such that $J^{*}(\lambda)=J(\lambda)$ for all $\lambda>0$, then $J^{*}(\lambda)$ is defined to be the analytic Wiener integral of $F$ over $C_{0}[0, T]$ with parameter $\lambda$ and, for $\lambda \in \mathbb{C}_{+}$, we write

$$
\int_{C_{0}[0, T]}^{a n w_{\lambda}} F(x) m(d x)=J^{*}(\lambda) .
$$

Let $q \neq 0$ be a real number and let $F$ be a functional such that $\int_{C_{0}[0, T]}^{a n w_{\lambda}} F(x) m(d x)$ exists for all $\lambda \in \mathbb{C}_{+}$. If the following limit exists, we call it the analytic Feynman integral of $F$ with parameter $q$ and we write

$$
\int_{C_{0}[0, T]}^{a n f_{q}} F(x) m(d x)=\lim _{\lambda \rightarrow-i q} \int_{C_{0}[0, T]}^{a n w_{\lambda}} F(x) m(d x),
$$

where $\lambda \longrightarrow-i q$ through $\mathbb{C}_{+}$.

\section{NOTATION.}

(i) For $\lambda \in \mathbb{C}_{+}$and $y \in C_{0}[0, T]$, let

$$
T_{\lambda}(F)(y)=\int_{C_{0}[0, T]}^{a n w_{\lambda}} F(x+y) m(d x) .
$$

(ii) Given a number $p$ with $1 \leq p \leq+\infty, p$ and $p^{\prime}$ are always related by $1 / p+$ $1 / p^{\prime}=1$.

(iii) Let $1<p \leq 2$ and let $\left\{H_{n}\right\}$ and $H$ be scale-invariant measurable functions such that for each $\rho>0$,

$$
\lim _{n \rightarrow \infty} \int_{C_{0}[0, T]}\left|H_{n}(\rho y)-H(\rho y)\right|^{p^{\prime}} m(d y)=0 .
$$

Then, we write

$$
\underset{n \rightarrow \infty}{\lim } .\left(w_{s}^{p^{\prime}}\right)\left(H_{n}\right) \approx H
$$

and we call $H$ the scale invariant limit in the mean of order $p^{\prime}$. A similar definition is understood when $n$ is replaced by the continuously varying parameter $\lambda$.

We are finally ready to state the definition of the $L_{p}$ analytic Fourier-Feynman transform [11], the definition of the convolution product [7], and the definition of the first variation of a function $[2,5]$.

Definition. Let $q \neq 0$ be a real number. For $1<p \leq 2$, we define the $L_{p}$ analytic Fourier-Feynman transform $T_{q}(p ; F)$ of $F$ by the formula $\left(\lambda \in \mathbb{C}_{+}\right)$ 


$$
T_{q}(p ; F)=\underset{\lambda \rightarrow-i q}{\lim . m_{s}}\left(w_{s}^{p^{\prime}}\right)\left(T_{\lambda}(F)\right)
$$

whenever this limit exists. Also, the $L_{1}$ analytic Fourier-Feynman transform $T_{\mathcal{q}}(1 ; F)$ of $F$ is defined by $\left(\lambda \in \mathbb{C}_{+}\right)$

$$
T_{\mathcal{q}}(1 ; F)=\lim _{\lambda \rightarrow-i q} T_{\lambda}(F) \quad \text { s-a.e. }
$$

We note that for $1 \leq p \leq 2, T_{q}(p ; F)$ is defined only s-a.e. We also note that if $T_{q}(p ; F)$ exists and if $F \approx G$, then $T_{q}(p ; G)$ exists and $T_{q}(p ; F) \approx T_{q}(p ; G)$.

Definition. Let $F$ and $G$ be functionals on $C_{0}[0, T]$. For $\lambda \in \mathbb{C}_{+}^{\sim}$, we define their convolution product (if it exists) by

$$
(F * G)_{\lambda}(y)=\left\{\begin{array}{l}
\int_{C_{0}[0, T]}^{a n w_{\lambda}} F\left(\frac{y+x}{\sqrt{2}}\right) G\left(\frac{y-x}{\sqrt{2}}\right) m(d x), \quad \lambda \in \mathbb{C}_{+} \\
\int_{\mathcal{C}_{0}[0, T]}^{a n f_{q}} F\left(\frac{y+x}{\sqrt{2}}\right) G\left(\frac{y-x}{\sqrt{2}}\right) m(d x), \quad \lambda=-i q, q \in \mathbb{R}, q \neq 0 .
\end{array}\right.
$$

\section{REMARKS.}

(i) When $\lambda=-i q$, we denote $(F * G)_{\lambda}$ by $(F * G)_{q}$.

(ii) Our definition of the convolution product is different from the definition given by Yeh in [14] and used by Yoo in [15]. In [14, 15], Yeh and Yoo studied the relationship between their convolution product and Fourier-Wiener transforms.

Next, we give the definition of the first variation $\delta F$ of a functional $F$.

Definition. Let $F$ be a Wiener measurable functional on $C_{0}[0, T]$ and let $w \in$ $C_{0}[0, T]$. Then

$$
\delta F(x \mid w)=\left.\frac{\partial}{\partial h} F(x+h w)\right|_{h=0}
$$

(if it exists) is called the first variation of $F(x)$.

We finish this section by describing the class of functionals that we work with in this paper. Let $n$ be a positive integer (fixed throughout this paper). Let $\left\{\alpha_{1}, \ldots, \alpha_{n}\right\}$ be an orthonormal set of functions in $L_{2}[0, T]$ and, for $\alpha, \beta \in L_{2}[0, T]$, let $(\alpha, \beta)=$ $\int_{0}^{T} \alpha(t) \beta(t) d t$. Also, for $x \in C_{0}[0, T]$ and $\alpha \in L_{2}[0, T]$, let $\langle\alpha, x\rangle$ denote the PaleyWiener-Zygmund stochastic integral $\int_{0}^{T} \alpha(t) d x(t)$. Let $m$ be a nonnegative integer. Then, for $1 \leq p<\infty$, let $B(p ; m)$ be the space of all functionals of the form

$$
F(x)=f\left(\left\langle\alpha_{1}, x\right\rangle, \ldots,\left\langle\alpha_{n}, x\right\rangle\right)
$$

for s-a.e. $x \in C_{0}[0, T]$, where all the $k$ th order partial derivatives of $f: \mathbb{R}^{n} \rightarrow \mathbb{R}$ are continuous and in $L_{p}\left(\mathbb{R}^{n}\right)$ for $k=0, \ldots, m$. Also, let $B(\infty ; m)$ be the space of all functionals of the form (2.11), where all the $k$ th order partial derivatives of $f$ are in $C_{0}\left(\mathbb{R}^{n}\right)$, the space of bounded continuous functions on $\mathbb{R}^{n}$ that vanish at infinity, for $k=0,1, \ldots, m$. Note that $B(p ; m+1) \subseteq B(p ; m)$ for $m=0,1, \ldots$. Finally, let

$$
A=\left\{y \in C_{0}[0, T]: y \text { is absolutely continuous on }[0, T] \text { with } y^{\prime} \in L_{2}[0, T]\right\} .
$$

3. Relationships involving two concepts. In [7], several relationships involving the Fourier-Feynman transform and the convolution product were established for 
functionals in $B(p ; 0)$. In this section, we also study relationships involving the first variation. In our first lemma, which follows easily from the definitions of $\delta F(x \mid w)$ and $B(p ; m)$, we obtain a formula for the first variation of functionals in $B(p ; m)$.

LEMMA 3.1. Let $p \in[1,+\infty], w \in A$ and $m \in \mathbb{N}$ be given. Let $F \in B(p ; m)$ be given by (2.11). Then

$$
\delta F(x \mid w)=\sum_{j=1}^{n}\left\langle\alpha_{j}, w\right\rangle f_{j}\left(\left\langle\alpha_{1}, x\right\rangle, \ldots,\left\langle\alpha_{n}, x\right\rangle\right)
$$

for s-a.e. $x \in C_{0}[0, T]$. Furthermore, as a function of $x, \delta F(x \mid w)$ is an element of $B(p ; m-1)$.

COROLLARY 3.1. Let $p, m$ and $F$ be as in Lemma 3.1. Let $w(t)=\int_{0}^{t} \alpha_{j}(s) d s$ on $[0, T]$ for some $j \in\{1, \ldots, n\}$. Then

$$
\delta F(x \mid w)=f_{j}\left(\left\langle\alpha_{1}, x\right\rangle, \ldots,\left\langle\alpha_{n}, x\right\rangle\right)
$$

for s-a.e. $x \in C_{0}[0, T]$.

COROLLARY 3.2. Let $p, m$ and $F$ be as in Lemma 3.1 and assume that $\left\{\alpha_{1}, \ldots, \alpha_{n}, w^{\prime}\right\}$ are orthogonal with $w \in A$. Then $\delta F(x \mid w)=0$ for s-a.e. $x \in C_{0}[0, T]$.

Our next corollary to Lemma 3.1 gives us a formula for $\delta^{l} F$.

Corollary 3.3. Let $m \in\{2,3, \ldots\}$ and let $l \in\{2, \ldots, m\}$. Let $p$ and $F$ be as in Lemma 3.1 and let $w_{1}, \ldots, w_{l}$ be elements of $A$. Then

$$
\begin{aligned}
\delta^{l} F\left(\cdot \mid w_{1}\right)\left(\cdot \mid w_{2}\right) \cdots\left(\cdot \mid w_{l-1}\right)\left(x \mid w_{l}\right) & \\
= & \sum_{j_{1}=1}^{n} \cdots \sum_{j_{l}=1}^{n}\left[\prod_{i=1}^{l}\left\langle\alpha_{j_{i}}, w_{i}\right\rangle\right] f_{j_{1}, \ldots, j_{l}}\left(\left\langle\alpha_{1}, x\right\rangle, \ldots,\left\langle\alpha_{n}, x\right\rangle\right)
\end{aligned}
$$

for s-a.e. $x \in C_{0}[0, T]$. Furthermore, $\delta^{l} F\left(\cdot \mid w_{1}\right) \cdots\left(\cdot \mid w_{l-1}\right)\left(x \mid w_{l}\right)$, as a function of $x$, is an element of $B(p ; m-l)$.

NotATION. For $\vec{u}=\left(u_{1}, \ldots, u_{n}\right) \in \mathbb{R}^{n}$, we write:

$$
\begin{aligned}
f(\vec{u}) & =f\left(u_{1}, \ldots, u_{n}\right), \\
f(\vec{u}+\langle\vec{\alpha}, y\rangle) & =f\left(u_{1}+\left\langle\vec{\alpha}_{1}, y\right\rangle, \ldots, u_{n}+\left\langle\alpha_{n}, y\right\rangle\right),
\end{aligned}
$$

and

$$
\|\vec{u}\|^{2}=\|\vec{u}\|_{2}^{2}=u_{1}^{2}+\cdots+u_{n}^{2} .
$$

In [7, Sec. 2], it was shown that $T_{q}(p ; F)$ exists for each $p \in[1,2]$, each $F \in B(p ; 0)$, and each nonzero $q \in \mathbb{R}$. In addition,

$$
\begin{aligned}
T_{\mathcal{q}}(p ; F)(y) & \approx\left(-\frac{i q}{2 \pi}\right)^{n / 2} \int_{\mathbb{R}^{n}} f(\vec{u}) \exp \left\{\frac{i q}{2}\|\vec{u}-\langle\vec{\alpha}, y\rangle\|^{2}\right\} d \vec{u} \\
& =\left(-\frac{i q}{2 \pi}\right)^{n / 2} \int_{\mathbb{R}^{n}} f(\vec{u}+\langle\vec{\alpha}, y\rangle) \exp \left\{\frac{i q}{2}\|\vec{u}\|^{2}\right\} d \vec{u} .
\end{aligned}
$$


Furthermore, $T_{q}(p ; F)$ is an element of $B\left(p^{\prime} ; 0\right)$, where $(1 / p)+\left(1 / p^{\prime}\right)=1$. Next, let $m \in \mathbb{N}$ and $F \in B(p ; m)$ be given. Since $B(p ; m) \subseteq B(p ; 0)$, we know that $T_{q}(p ; F)$ exists and is given by (3.7). The proof that $T_{q}(p ; F)$ belongs to $B\left(p^{\prime} ; m\right)$ for $m>0$ is similar to the proof given in [7] for the case $m=0$.

In our first theorem, we show that the transform with respect to the first argument of the variation equals the variation of the transform.

TheOrem 3.1. Let $p \in[1,2]$, let $m \in\{1,2, \ldots\}$, let $F \in B(p ; m)$ be given by (2.11), and let $w \in A$. Then, for all real $q \neq 0$ and s-a.e. $y \in C_{0}[0, T]$,

$$
T_{q}(p ; \delta F(\cdot \mid w))(y)=\delta T_{q}(p ; F)(y \mid w) .
$$

Also, both of the expressions in (3.8) are given by the expression

$$
\left(-\frac{i q}{2 \pi}\right)^{n / 2} \int_{\mathbb{R}^{n}}\left[\sum_{j=1}^{n}\left\langle\alpha_{j}, w\right\rangle f_{j}(\vec{u}+\langle\vec{\alpha}, y\rangle)\right] \exp \left\{\frac{i q}{2}\|\vec{u}\|^{2}\right\} d \vec{u},
$$

which, as a function of $y$, is a element of $B\left(p^{\prime} ; m-1\right)$.

Proof. First, using the definition of the first variation and equation (3.7), we see that

$$
\begin{aligned}
\delta T_{q}(p ; F)(y \mid w) & =\left.\frac{\partial}{\partial h}\left[T_{q}(p ; F)(y+h w)\right]\right|_{h=0} \\
& =\left(-\frac{i q}{2 \pi}\right)^{n / 2} \int_{\mathbb{R}^{n}}\left[\sum_{j=1}^{n}\left\langle\alpha_{j}, w\right\rangle f_{j}(\vec{u}+\langle\vec{\alpha}, y\rangle)\right] \exp \left\{\frac{i q}{2}\|\vec{u}\|^{2}\right\} d \vec{u} .
\end{aligned}
$$

Next, using equation (3.1), we see that

$$
\begin{aligned}
T_{q}(p ; \delta F(\cdot \mid w))(y) & =\int_{C_{0}[0, T]}^{a n f_{q}} \delta F(x+y \mid w) m(d x) \\
& =\int_{C_{0}[0, T]}^{a n f_{q}} \sum_{j=1}^{n}\left\langle\alpha_{j}, w\right\rangle f_{j}(\langle\vec{\alpha}, x+y\rangle) m(d x) .
\end{aligned}
$$

Then, evaluating the above analytic Feynman integral, we obtain (3.9) as desired. Finally, $\delta T_{q}(p ; F)(y \mid w)$ is an element of $B\left(p^{\prime} ; m-1\right)$ since $T_{q}(p ; F)$ is an element of $B\left(p^{\prime} ; m\right)$.

Next, taking further variations of the expression given in (3.9), we obtain the following corollary.

Corollary 3.4. Let $p \in[1,2], m \in\{2,3, \ldots\}$ and $l \in\{2, \ldots, m\}$ be given. Let $F \in$ $B(p ; m)$ be given by (2.11), and let $w_{1}, \ldots, w_{l}$ be in $A$. Then

$$
\begin{aligned}
& \delta^{l} T_{q}(p ; F)\left(\cdot \mid w_{1}\right) \cdots\left(\cdot \mid w_{l-1}\right)\left(y \mid w_{l}\right) \\
& =\left(-\frac{i q}{2 \pi}\right)^{n / 2} \int_{\mathbb{R}^{n}} \sum_{j_{1}=1}^{n} \cdots \sum_{j_{l}=1}^{n}\left[\prod_{i=1}^{l}\left\langle\alpha_{j_{i}}, w_{i}\right\rangle\right] f_{j_{1}, \ldots, j_{l}}(\vec{u}+\langle\vec{\alpha}, y\rangle) \exp \left\{\frac{i q}{2}\|\vec{u}\|^{2}\right\} d \vec{u},
\end{aligned}
$$

which, as a function of $y$, is an element of $B\left(p^{\prime} ; m-l\right)$. 
In our next theorem, we show that the transform with respect to the second argument of the variation equals the variation of the functional.

THEOREM 3.2. Let $p, q, w$, and $F$ be as in Theorem 3.1. Then, for $s$-a.e. $y \in C_{0}[0, T]$,

$$
T_{q}(p ; \delta F(y \mid \cdot))(w)=\delta F(y \mid w),
$$

which, as a function of $y$, is an element of $B(p ; m-1)$.

Proof. Using equation (3.1), we see that

$$
\begin{aligned}
T_{q}(p ; \delta F(y \mid \cdot))(w) & =\int_{C_{0}[0, T]}^{a n f_{q}} \delta F(y \mid w+x) m(d x) \\
& =\int_{C_{0}[0, T]}^{a n f_{q}} \sum_{j=1}^{n}\left\langle\alpha_{j}, w+x\right\rangle f_{j}(\langle\vec{\alpha}, y\rangle) m(d x) \\
& =\sum_{j=1}^{n} f_{j}(\langle\vec{\alpha}, y\rangle) \int_{C_{0}[0, T]}^{a n f_{q}}\left\langle\alpha_{j}, w+x\right\rangle m(d x) \\
& =\sum_{j=1}^{n} f_{j}(\langle\vec{\alpha}, y\rangle)\left[\left\langle\alpha_{j}, w\right\rangle+0\right] \\
& =\delta F(y \mid w)
\end{aligned}
$$

for s-a.e. $y \in C_{0}[0, T]$.

Our next lemma involves the convolution product of functionals from various $B(p ; m)$ classes.

LEMmA 3.2. Let $p, m, F$, and $q$ be as in Theorem 3.1. Let $G \in B\left(p^{\prime} ; m\right)$ be given by

$$
G(x)=g\left(\left\langle\alpha_{1}, x\right\rangle, \ldots,\left\langle\alpha_{n}, x\right\rangle\right)=g(\langle\vec{\alpha}, x\rangle)
$$

for s-a.e. $x \in C_{0}[0, T]$. Then $(F * G)_{q}$ is an element of $B(\infty ; m)$.

Proof. First note that, for s-a.e. $y \in C_{0}[0, T]$,

$$
(F * G)_{q}(y)=l(\langle\vec{\alpha}, y\rangle),
$$

where

$$
l(\vec{v})=\left(-\frac{i q}{2 \pi}\right)^{n / 2} \int_{\mathbb{R}^{n}} f\left(\frac{\vec{v}+\vec{u}}{\sqrt{2}}\right) g\left(\frac{\vec{v}-\vec{u}}{\sqrt{2}}\right) \exp \left\{\frac{i q}{2}\|\vec{u}\|^{2}\right\} d \vec{u} .
$$

Hence, for each $\vec{v} \in \mathbb{R}^{n}$,

$$
\begin{aligned}
|l(\vec{v})| & \leq\left|\frac{q}{2 \pi}\right|^{n / 2} \int_{\mathbb{R}^{n}}\left|f\left(\frac{\vec{v}+\vec{u}}{\sqrt{2}}\right) g\left(\frac{\vec{v}-\vec{u}}{\sqrt{2}}\right)\right| d \vec{u} \\
& \leq\left|\frac{q}{2 \pi}\right|^{n / 2}\left\{\int_{\mathbb{R}^{n}}\left|f\left(\frac{\vec{v}+\vec{u}}{\sqrt{2}}\right)\right|^{p} d \vec{u}\right\}^{1 / p}\left\{\int_{\mathbb{R}^{n}}\left|g\left(\frac{\vec{v}-\vec{u}}{\sqrt{2}}\right)\right|^{p^{\prime}} d \vec{u}\right\}^{1 / p^{\prime}} \\
& =\left|\frac{q}{2 \pi}\right|^{n / 2}\|f\|_{p}\|g\|_{p^{\prime}} .
\end{aligned}
$$

Now, a standard argument shows that $l$ belongs to $C_{0}\left(\mathbb{R}^{n}\right)$. Hence, $(F * G)_{q}$ is an element of $B(\infty ; m)$. 
In our next theorem, we obtain a formula for the first variation of the convolution product.

THEOREM 3.3. Let $p, m, q, F$, and $G$ be as in Lemma 3.2, and let $w \in A$. Then for s-a.e. $y \in C_{0}[0, T], \delta(F * G)_{q}(y \mid w)$ exists and is given by the last expression in equation (3.19) below. Furthermore, as a function of $y, \delta(F * G)_{q}(y \mid w)$ is an element of $B(\infty ; m-1)$.

Proof. By Lemma 3.2, $(F * G)_{q}$ is an element of $B(\infty ; m)$ and so, by Lemma 3.1, $\delta(F * G)_{q}(y \mid w)$ is an element of $B(\infty ; m-1)$. Furthermore,

$$
\begin{aligned}
\delta(F * G)_{q}(y \mid w) & =\left.\frac{\partial}{\partial h}\left[(F * G)_{q}(y+h w)\right]\right|_{h=0} \\
= & \left.\frac{\partial}{\partial h}\left[\int_{C_{0}[0, T]}^{a n f_{q}} F\left(\frac{y+h w+x}{\sqrt{2}}\right) G\left(\frac{y+h w-x}{\sqrt{2}}\right) m(d x)\right]\right|_{h=0} \\
= & \frac{\partial}{\partial h}\left[\left(-\frac{i q}{2 \pi}\right)^{n / 2} \int_{\mathbb{R}^{n}} f\left(\frac{\langle\vec{\alpha}, y+h w\rangle+\vec{u}}{\sqrt{2}}\right) g\left(\frac{\langle\vec{\alpha}, y+h w\rangle-\vec{u}}{\sqrt{2}}\right)\right. \\
= & \left(-\frac{i q}{2 \pi}\right)^{n / 2} \int_{\mathbb{R}^{n}}\left[f\left(\frac{\langle\vec{\alpha}, y\rangle+\vec{u}}{\sqrt{2}}\right) \sum_{j=1}^{n}\left\langle\alpha_{j}, w / \sqrt{2}\right\rangle g_{j}\left(\frac{\langle\vec{\alpha}, y\rangle-\vec{u}}{\sqrt{2}}\right)\right. \\
& \left.+g\left(\frac{\langle\vec{\alpha}, y\rangle-\vec{u}}{\sqrt{2}}\right) \sum_{j=1}^{n}\left\langle\alpha_{j}, w / \sqrt{2}\right\rangle f_{j}\left(\frac{\langle\vec{\alpha}, y\rangle+\vec{u}}{\sqrt{2}}\right)\right] \exp \left\{\frac{i q}{2}\|\vec{u}\|^{2}\right\} d \vec{u} .
\end{aligned}
$$

Next, we obtain formulas for the convolution product of the first variation of functionals. In Theorem 3.4, we take the convolution with respect to the first argument of the variations while in Theorem 3.5, we take the convolution with respect to the second argument of the variations.

THEOREM 3.4. Let $p, m, q, w, F$, and $G$ be as in Theorem 3.3. Then, for s-a.e. $y \in$ $C_{0}[0, T],\left(\delta F(\cdot \mid w) * \delta G(\cdot \mid w)_{q}\right)(y)$ exists and is given by the last expression in equation (3.20) below. Furthermore, as a function of $y,(\delta F(\cdot \mid w) * \delta G(\cdot \mid w))_{q}(y)$ is an element of $B(\infty ; m-1)$.

Proof. Since $F \in B(p ; m)$ and $G \in B\left(p^{\prime} ; m\right)$, it follows, from Lemma 3.1, that $\delta F(y \mid w) \in B(p ; m-1)$ and $\delta G(y \mid w) \in B\left(p^{\prime} ; m-1\right)$. Hence, by Lemma 3.2, $(\delta F(\cdot \mid$ $w) * \delta G(\cdot \mid w))_{q}(y)$ is an element of $B(\infty ; m-1)$. Also, by equations (2.9) and (3.1),

$$
\begin{aligned}
& (\delta F(\cdot \mid w) * \delta G(\cdot \mid w))_{q}(y) \\
& \quad=\int_{C_{0}[0, T]}^{a n f_{q}} \delta F\left(\frac{y+x}{\sqrt{2}} \mid w\right) \delta G\left(\frac{y-x}{\sqrt{2}} \mid w\right) m(d x) \\
& \quad=\int_{C_{0}[0, T]}^{a n f_{q}}\left[\sum_{j=1}^{n}\left\langle\alpha_{j}, w\right\rangle f_{j}\left(\left\langle\vec{\alpha}, \frac{y+x}{\sqrt{2}}\right\rangle\right)\right]\left[\sum_{k=1}^{n}\left\langle\alpha_{k}, w\right\rangle g_{k}\left(\left\langle\vec{\alpha}, \frac{y-x}{\sqrt{2}}\right\rangle\right)\right] m(d x)
\end{aligned}
$$




$$
\begin{gathered}
=\left(-\frac{i q}{2 \pi}\right)^{n / 2} \int_{\mathbb{R}^{n}} \exp \left\{\frac{i q}{2}\|\vec{u}\|^{2}\right\}\left[\sum_{j=1}^{n}\left\langle\alpha_{j}, w\right\rangle f_{j}\left(\frac{\langle\vec{\alpha}, y\rangle+\vec{u}}{\sqrt{2}}\right)\right] \\
\cdot\left[\sum_{k=1}^{n}\left\langle\alpha_{k}, w\right\rangle g_{k}\left(\frac{\langle\vec{\alpha}, y\rangle-\vec{u}}{\sqrt{2}}\right)\right] d \vec{u} .
\end{gathered}
$$

THEOREM 3.5. Let $p, m, w, F, G$, and $q$ be as in Theorem 3.3. Then, for s-a.e. $y \in$ $C_{0}[0, T],(\delta F(y \mid \cdot) * \delta G(y \mid \cdot))_{q}(w)$ exists and is given by the formula

$$
\begin{aligned}
& (\delta F(y \mid \cdot) * \delta G(y \mid \cdot))_{q}(w) \\
& \quad=\delta F(y \mid w / \sqrt{2}) \delta G(y \mid w / \sqrt{2})-\frac{i}{2 q} \sum_{j=1}^{n} f_{j}(\langle\vec{\alpha}, y\rangle) g_{j}(\langle\vec{\alpha}, y\rangle) .
\end{aligned}
$$

Furthermore, as a function of $y,(\delta F(y \mid \cdot) * \delta G(y \mid \cdot))_{q}(w)$ is an element of $B(1$; $m-1)$.

Proof. The conclusions of this theorem follow from the calculations below:

$$
\begin{aligned}
&(\delta F(y \mid \cdot) * \delta G(y \mid \cdot))_{q}(w) \\
& \quad=\int_{C_{0}[0, T]}^{a n f_{q}} \delta F\left(y \mid \frac{w+x}{\sqrt{2}}\right) \delta G\left(y \mid \frac{w-x}{\sqrt{2}}\right) m(d x) \\
&=\int_{C_{0}[0, T]}^{a n f_{q}}\left[\sum_{j=1}^{n} \frac{\left\langle\alpha_{j}, w+x\right\rangle}{\sqrt{2}} f_{j}(\langle\vec{\alpha}, y\rangle)\right]\left[\sum_{k=1}^{n} \frac{\left\langle\alpha_{k}, w-x\right\rangle}{\sqrt{2}} g_{k}(\langle\vec{\alpha}, y\rangle)\right] m(d x) \\
&=\sum_{j=1}^{n} \sum_{k=1}^{n} f_{j}(\langle\vec{\alpha}, y\rangle) g_{k}(\langle\vec{\alpha}, y\rangle) \cdot \frac{1}{2} \int_{C_{0}[0, T]}^{a n f_{q}}\left\langle\alpha_{j}, w+x\right\rangle\left\langle\alpha_{k}, w-x\right\rangle m(d x) \\
&=\sum_{j=1}^{n} \sum_{k=1}^{n} f_{j}(\langle\vec{\alpha}, y\rangle) g_{k}(\langle\vec{\alpha}, y\rangle) \cdot \frac{1}{2}\left[\left\langle\alpha_{j}, w\right\rangle\left\langle\alpha_{k}, w\right\rangle-(i / q)\left(\alpha_{j}, \alpha_{k}\right)\right] \\
&= \delta F(y \mid w / \sqrt{2}) \delta G(y \mid w / \sqrt{2})-\frac{i}{2 q} \sum_{j=1}^{n} f_{j}(\langle\vec{\alpha}, y\rangle) g_{j}(\langle\vec{\alpha}, y\rangle)
\end{aligned}
$$

since

$$
\int_{C_{0}[0, T]}^{a n f_{q}}\left\langle\alpha_{j}, x\right\rangle\left\langle\alpha_{k}, x\right\rangle m(d x)=\frac{i}{q}\left(\alpha_{j}, \alpha_{k}\right) \text { and } \int_{C_{0}[0, T]}^{a n f_{q}}\left\langle\alpha_{j}, x\right\rangle m(d x)=0 .
$$

We conclude this section with two theorems relating transforms and convolutions. Recall that the analytic Fourier-Feynman integral $T_{q}(p ; F)$ is defined only for $p \in[1,2]$.

TheOrem 3.6. Let $m \in\{0,1,2, \ldots\}, F \in B(2 ; m)$ be given by (2.11) and $G \in B(2 ; m)$ be given by (3.15). Then, for all real $q \neq 0,\left(T_{q}(2 ; F) * T_{q}(2 ; G)\right)_{q}$ exists as an element of $B(\infty ; m)$ and for s-a.e. $y \in C_{0}[0, T]$,

$$
\begin{array}{rl}
\left(T_{q}(2 ; F) * T_{q}(2 ; G)\right)_{q}(y)=\left(-\frac{i q}{2 \pi}\right)^{3 n / 2} \int_{\mathbb{R}^{3 n}} & f\left(\vec{u}+\frac{\langle\vec{\alpha}, y\rangle+\vec{r}}{\sqrt{2}}\right) g\left(\vec{v}+\frac{\langle\vec{\alpha}, y\rangle-\vec{r}}{\sqrt{2}}\right) \\
\cdot \exp \left\{\frac{i q}{2}\left(\|\vec{u}\|^{2}+\|\vec{v}\|^{2}+\|\vec{r}\|^{2}\right)\right\} d \vec{u} d \vec{v} d \vec{r}
\end{array}
$$


Proof. First, we note, by the discussion following Corollary 3.3, that $T_{q}(2 ; F)$ and $T_{q}(2 ; G)$ both exist and are elements of $B(2 ; m)$. Hence, $\left(T_{q}(2 ; F) * T_{q}(2 ; G)\right)_{q}$ is an element of $B(\infty ; m)$. Equation (3.24) then follows upon the evaluation of the analytic Feynman integral

$$
\int_{C_{0}[0, T]}^{a n f_{q}} T_{q}(2 ; F)\left(\frac{y+x}{\sqrt{2}}\right) T_{q}(2 ; G)\left(\frac{y-x}{\sqrt{2}}\right) m(d x) .
$$

ThEOREM 3.7. Let $m \in\{0,1,2, \ldots\}, F \in B\left(p_{1} ; m\right)$, and $G \in B\left(p_{2} ; m\right)$ with $1 \leq p_{1} \leq$ $2,1 \leq p_{2} \leq 2$ and $\left(1 / p_{1}\right)+\left(1 / p_{2}\right) \geq(3 / 2)$. Let $r$ be given by $(1 / r)=\left(1 / p_{1}\right)+\left(1 / p_{2}\right)-1$. Then $(F * G)_{q} \in B(r ; m)$ and, for s-a.e. $y \in C_{0}[0, T]$,

$$
T_{q}\left(r ;(F * G)_{q}\right)(y)=T_{q}\left(p_{1} ; F\right)(y / \sqrt{2}) T_{q}\left(p_{2} ; G\right)(y / \sqrt{2}) .
$$

Proof. First, we note that $r \in[1,2]$. For the case $m=0$, it was shown, in [10, p. 29], that $(F * G)_{q} \in B(r ; 0)$ and that equation (3.26) is valid. But, since $B(p ; m+1) \subseteq$ $B(p ; m)$ for all $m \in\{0,1,2, \ldots\}$ and all $p \in[1,+\infty]$, we see that $(F * G)_{q} \in B(r ; m)$ and that equation (3.26) is valid for all $m \in\{0,1,2, \ldots\}$.

By choosing specific values for $p_{1}$ and $p_{2}$ in Theorem 3.7, we obtain the following corollary.

COROLlary 3.5. Let $m \in\{0,1,2, \ldots\}$.

(i) If $F, G \in B(1 ; m)$, then $(F * G)_{q} \in B(1 ; m)$ and, for s-a.e. $y \in C_{0}[0, T]$,

$$
T_{q}\left(1 ;(F * G)_{q}\right)(y)=T_{q}(1 ; F)(y / \sqrt{2}) T_{q}(1 ; G)(y / \sqrt{2}) .
$$

(ii) If $F \in B(1 ; m)$ and $G \in B(2 ; m)$, then $(F * G)_{q} \in B(2 ; m)$ and, for s-a.e. $y \in$ $C_{0}[0, T]$,

$$
T_{q}\left(2 ;(F * G)_{q}\right)(y)=T_{q}(1 ; F)(y / \sqrt{2}) T_{q}(2 ; G)(y / \sqrt{2}) .
$$

(iii) If $F, G \in B(4 / 3 ; m)$, then $(F * G)_{q} \in B(2 ; m)$ and, for s-a.e. $y \in C_{0}[0, T]$,

$$
T_{q}\left(2 ;(F * G)_{q}\right)(y)=T_{q}(4 / 3 ; F)(y / \sqrt{2}) T_{q}(4 / 3 ; G)(y / \sqrt{2}) .
$$

4. Relationships involving three concepts. In this section, we look at all the relationships involving the "transform," the "convolution," and "variation" where each operation is used exactly once. There are more than six possibilities since one can take both the transform and the convolution with respect to the first or the second argument of the variation. However, there are some repetitions, for example, we observed, in Theorem 3.2, that the transform with respect to the second argument of the variation equals the variation of the functional. It turns out that there are nine distinct possibilities. We state formally five of these results as theorems (namely, Theorem 4.1 through Theorem 4.5 ) and the other four results as formulas (namely, equation (4.11) through equation (4.14)). However, all nine of these results hold for s-a.e. $y \in C_{0}[0, T]$ and all real $q \neq 0$.

In our first theorem, we obtain a formula for the transform with respect to the first argument of the variation of the convolution product. 
THEOREM 4.1. Let $m$ be a positive integer, let $w \in A$, and let $p_{1}, p_{2}, r, F$, and $G$ be as in Theorem 3.7. Then

$$
\begin{aligned}
T_{q}\left(r ; \delta(F * G)_{q}(\cdot \mid w)\right)(y)= & \delta T_{q}\left(r ;(F * G)_{q}\right)(y \mid w) \\
= & T_{q}\left(p_{1} ; F\right)(y / \sqrt{2}) \delta T_{q}\left(p_{2} ; G\right)(y / \sqrt{2} \mid w / \sqrt{2}) \\
& +\delta T_{q}\left(p_{1} ; F\right)(y / \sqrt{2} \mid w / \sqrt{2}) T_{q}\left(p_{2} ; G\right)(y / \sqrt{2}),
\end{aligned}
$$

which, as a function of $y$, is an element of $B\left(r^{\prime} ; m-1\right)$.

Proof. The first equality in (4.1) follows from (3.8). But, by Theorem 3.7, $(F * G)_{q} \in$ $B(r ; m)$ and so, using (3.26), we see that

$$
\begin{aligned}
\delta T_{q}\left(r ;(F * G)_{q}\right)(y \mid w) & =\left.\frac{\partial}{\partial h}\left[T_{q}\left(r ;(F * G)_{q}\right)(y+h w)\right]\right|_{h=0} \\
& =\left.\frac{\partial}{\partial h}\left[T_{q}\left(p_{1} ; F\right)\left(\frac{y+h w}{\sqrt{2}}\right) T_{q}\left(p_{2} ; G\right)\left(\frac{y+h w}{\sqrt{2}}\right)\right]\right|_{h=0}
\end{aligned}
$$

which equals the last expression on the right-hand side of equation (4.1).

REMARK. By choosing specific values for $p_{1}$ and $p_{2}$ in Theorem 4.1 (as we did in Corollary 3.5 ), one gets various versions of equation (4.1). For example, if $p_{1}=p_{2}=$ $4 / 3$, then $r=r^{\prime}=2$ and so, using (3.8) and (3.29), we see that

$$
\begin{aligned}
T_{\mathcal{q}}\left(2 ; \delta(F * G)_{q}(\cdot \mid w)\right)(y)= & \delta T_{q}\left(2 ;(F * G)_{q}\right)(y \mid w) \\
= & T_{q}(4 / 3 ; F)(y / \sqrt{2}) \delta T_{q}(4 / 3 ; G)(y / \sqrt{2} \mid w / \sqrt{2}) \\
& +\delta T_{q}(4 / 3 ; F)(y / \sqrt{2} \mid w / \sqrt{2}) T_{q}(4 / 3 ; G)(y / \sqrt{2}),
\end{aligned}
$$

which, as a function of $y$, is an element of $B(2 ; m-1)$.

Using Theorem 3.2 and Theorem 3.7, it follows that the transform with respect to the second argument of the variation of the convolution product equals the variation of the convolution product.

THEOREM 4.2. Let $m, w, p_{1}, p_{2}, r, F$, and $G$ be as in Theorem 4.1. Then $(F * G)_{q} \in$ $B(r ; m)$ and

$$
T_{q}\left(r ; \delta(F * G)_{q}(y \mid \cdot)\right)(w)=\delta(F * G)_{q}(y \mid w),
$$

which, as a function of $y$, is an element of $B(r ; m-1)$.

Next, we seek formulas for the transforms of the convolution product with respect to the first argument of the variations. Here, there are two cases, namely, we can take the transform of the expression

$$
(\delta F(\cdot \mid w) * \delta G(\cdot \mid w))_{q}(y)
$$

either with respect to $y$ (Theorem 4.3 below), or else with respect to $w$ (Theorem 4.4 below). 
THEOREM 4.3. Let $m, w, p_{1}, p_{2}, r, F$, and $G$ be as in Theorem 4.1. Then

$$
T_{q}\left(r ;(\delta F(\cdot \mid w) * \delta(\cdot \mid w))_{q}\right)(y)=\delta T_{q}\left(p_{1} ; F\right)(y / \sqrt{2} \mid w) \delta T_{q}\left(p_{2} ; G\right)(y / \sqrt{2} \mid w),
$$

which, as a function of $y$, is an element of $B\left(r^{\prime} ; m-1\right)$.

Proof. Equation (4.6) follows immediately from equations (3.26) and (3.8).

REMARK. Again, choosing specific values for $p_{1}$ and $p_{2}$ in Theorem 4.3 (as we did in Corollary 3.5), one gets various versions of equation (4.6).

THEOREM 4.4. Let $p, m, w, F$, and $G$ be as in Theorem 3.3. Then, the analytic FourierFeynman transform of the expressions in equation (3.20) with respect to $w$ is given by the expression

$$
\begin{aligned}
& (\delta F(\cdot \mid w) * \delta G(\cdot \mid w))_{q}(y) \\
+ & \left(\frac{i}{q}\right)\left(-\frac{i q}{2 \pi}\right)^{n / 2} \int_{\mathbb{R}^{n}}\left[\sum_{j=1}^{n} f_{j}\left(\frac{\langle\vec{\alpha}, y\rangle+\vec{u}}{\sqrt{2}}\right) g_{j}\left(\frac{\langle\vec{\alpha}, y\rangle-\vec{u}}{\sqrt{2}}\right)\right] \exp \left\{\frac{i q}{2}\|\vec{u}\|^{2}\right\} d \vec{u},
\end{aligned}
$$

which, as a function of $y$, is an element of $B(\infty ; m-1)$.

Proof. To obtain (4.7), we simply substitute the last expression in equation (3.20), with $w$ replaced with $w+x$, into the analytic Feynman integral

$$
\int_{C_{0}[0, T]}^{a_{n} f_{q}}(\delta F(\cdot \mid w+x) * \delta G(\cdot \mid w+x))_{q}(y) m(d x),
$$

and then evaluate this integral using (3.23).

Our next goal is to obtain formulas for the transforms of the convolution product with respect to the second argument of the variations. Again, there are two cases since we can take the transform of the expressions in equation (3.21) either with respect to $w$ (Theorem 4.5 below) or else with respect to $y$ (equation (4.11) below).

THEOREM 4.5. Let $p \in[1,2]$, let $m$ be a positive integer, let $F \in B(p ; m)$, and let $G \in B\left(p^{\prime} ; m\right)$. Then

$$
T_{q}\left(p ;(\delta F(y \mid \cdot) * \delta G(y \mid \cdot))_{q}\right)(w)=\delta F(y \mid w / \sqrt{2}) \delta G(y \mid w / \sqrt{2}),
$$

which, as a function of $y$, is an element of $B(1 ; m-1)$.

Proof. Using equation (3.21) and then equation (3.1), we obtain that the left-hand side of (4.9) equals the analytic Feynman integral

$$
\begin{gathered}
\int_{C_{0}[0, T]}^{a n f_{q}}\left[\delta F\left(y \mid \frac{w+x}{\sqrt{2}}\right) \delta G\left(y \mid \frac{w+x}{\sqrt{2}}\right)-\frac{i}{2 q} \sum_{j=1}^{n} f_{j}(\langle\vec{\alpha}, y\rangle) g_{j}(\langle\vec{\alpha}, y\rangle)\right] m(d x) \\
=\int_{C_{0}[0, T]}^{a n f_{q}}\left\{\left[\sum_{j=1}^{n}\left\langle\alpha_{j}, \frac{w+x}{\sqrt{2}}\right\rangle f_{j}(\langle\vec{\alpha}, y\rangle)\right]\left[\sum_{k=1}^{n}\left\langle\alpha_{k}, \frac{w+x}{\sqrt{2}}\right\rangle g_{k}(\langle\vec{\alpha}, y\rangle)\right]\right. \\
\left.-\frac{i}{2 q} \sum_{j=1}^{n} f_{j}(\langle\vec{\alpha}, y\rangle) g_{j}(\langle\vec{\alpha}, y\rangle)\right\} m(d x) .
\end{gathered}
$$


Using (3.23) to evaluate the above Feynman integral, yields the right-hand side of (4.9).

Under the hypotheses of Theorem 4.5, the transform of the expressions in equation (3.21) with respect to $y$ yields

$$
\begin{aligned}
\int_{C_{0}[0, T]}^{a n f_{q}}( & \delta F(y+x \mid \cdot) * \delta G(y+x \mid \cdot))_{q}(w) m(d x) \\
= & \int_{C_{0}[0, T]}^{a n f_{q}}[\delta F(y+x \mid w / \sqrt{2}) \delta G(y+x \mid w / \sqrt{2}) \\
& \left.-\frac{i}{2 q} \sum_{j=1}^{n} f_{j}(\langle\vec{\alpha}, y+x\rangle) g_{j}(\langle\vec{\alpha}, y+x\rangle)\right] m(d x) \\
= & \int_{C_{0}[0, T]}^{a n f_{q}}\left\{\left[\sum_{j=1}^{n}\left\langle\alpha_{j}, w / \sqrt{2}\right\rangle f_{j}(\langle\vec{\alpha}, y+x\rangle)\right]\left[\sum_{k=1}^{n}\left\langle\alpha_{k}, w / \sqrt{2}\right\rangle g_{k}(\langle\vec{\alpha}, y+x\rangle)\right]\right. \\
= & \left.-\frac{i}{2 q} \sum_{j=1}^{n} f_{j}(\langle\vec{\alpha}, y+x\rangle) g_{j}(\langle\vec{\alpha}, y+x\rangle)\right\} m(d x) \\
& \times \int_{\mathbb{R}^{n}} \exp \left\{\frac{i q}{2 \pi}\|\vec{u}\|^{2}\right\} \cdot f_{j}(\langle\vec{\alpha}, y\rangle+\vec{u}) g_{k}(\langle\vec{\alpha}, y\rangle+\vec{u}) d \vec{u} \\
& -\frac{i}{2 q}\left(-\frac{i q}{2 \pi}\right)^{n / 2} \sum_{j=1}^{n}\left\langle\alpha_{\mathbb{R}^{n}} f_{j}(\langle\vec{\alpha}, y\rangle+\vec{u}) g_{j}(\langle\vec{\alpha}, y\rangle+\vec{u}) \exp \left\{\frac{i q}{2}\|\vec{u}\|^{2}\right\} d \vec{u} .\right.
\end{aligned}
$$

Next, we want to take the variation of the expressions in equation (3.24). So, let $m$ be a positive integer, let $F$ and $G$ be elements of $B(2 ; m)$, and let $w \in A$. Then

$$
\begin{gathered}
\delta\left(T_{q}(2 ; F) * T_{q}(2 ; G)\right)_{q}(y \mid w) \\
=\left.\frac{\partial}{\partial h}\left[\left(T_{q}(2 ; F) * T_{q}(2 ; G)\right)_{q}(y+h w)\right]\right|_{h=0} \\
=\frac{\partial}{\partial h}\left[\left(-\frac{i q}{2 \pi}\right)^{3 n / 2} \int_{\mathbb{R}^{3 n}} f\left(\vec{u}+\frac{\langle\vec{\alpha}, y+h w\rangle+\vec{r}}{\sqrt{2}}\right) g\left(\vec{v}+\frac{\langle\vec{\alpha}, y+h w\rangle+\vec{r}}{\sqrt{2}}\right)\right. \\
\left.\cdot \exp \left\{\frac{i q}{2}\left(\|\vec{u}\|^{2}+\|\vec{v}\|^{2}+\|\vec{r}\|^{2}\right)\right\} d \vec{u} d \vec{v} d \vec{r}\right]\left.\right|_{h=0} \\
=\left(-\frac{i q}{2 \pi}\right)^{3 n / 2} \int_{\mathbb{R}^{3 n}}\left[f\left(\vec{u}+\frac{\langle\vec{\alpha}, y\rangle+\vec{r}}{\sqrt{2}}\right) \sum_{j=1}^{n}\left\langle\alpha_{j}, w / \sqrt{2}\right\rangle g_{j}\left(\vec{v}+\frac{\langle\vec{\alpha}, y\rangle+\vec{r}}{\sqrt{2}}\right)\right. \\
\left.+g\left(\vec{v}+\frac{\langle\vec{\alpha}, y\rangle+\vec{r}}{\sqrt{2}}\right) \sum_{j=1}^{n}\left\langle\alpha_{j}, w / \sqrt{2}\right\rangle f_{j}\left(\vec{u}+\frac{\langle\vec{\alpha}, y\rangle+\vec{r}}{\sqrt{2}}\right)\right] \\
\cdot \exp \left\{\frac{i q}{2}\left(\|\vec{u}\|^{2}+\|\vec{v}\|^{2}+\|\vec{r}\|^{2}\right)\right\} d \vec{u} d \vec{v} d \vec{r},
\end{gathered}
$$

which, as a function of $y$, is an element of $B(\infty ; m-1)$. 
We finish up this section by finding formulas for the convolution product of the transform of the variation. Again, there are two cases, namely, we can take the convolution with respect to the first argument (equation (4.13) below) or the second argument (equation (4.14) below) of the variation. However, in both cases, the transform is taken with respect to the first argument of the variation. So, let $m$ be a positive integer, let $F$ and $G$ be elements of $B(2 ; m)$, and let $w \in A$. Then

$$
\begin{aligned}
\left(\delta T_{q}(2 ; F)(\cdot \mid w) * \delta T_{q}(2 ; G)(\cdot \mid w)\right)_{q}(y) & \\
= & \int_{C_{0}[0, T]}^{a n f_{q}} \delta T_{q}(2 ; F)\left(\frac{y+x}{\sqrt{2}} \mid w\right) \delta T_{q}(2 ; G)\left(\frac{y-x}{\sqrt{2}} \mid w\right) m(d x) \\
= & \int_{C_{0}[0, T]}^{a n f_{q}}\left(-\frac{i q}{2 \pi}\right)^{n / 2} \int_{\mathbb{R}^{n}}\left[\sum_{j=1}^{n}\left\langle\alpha_{j}, w\right\rangle f_{j}(\vec{u}+\langle\vec{\alpha},(y+x) / \sqrt{2}\rangle)\right] \exp \left\{\frac{i q}{2}\|\vec{u}\|^{2}\right\} d \vec{u} \\
& \cdot\left(-\frac{i q}{2 \pi}\right)^{n / 2} \int_{\mathbb{R}^{n}}\left[\sum_{k=1}^{n}\left\langle\alpha_{k}, w\right\rangle g_{k}(\vec{v}+\langle\vec{\alpha},(y-x) / \sqrt{2}\rangle)\right] \exp \left\{\frac{i q}{2}\|\vec{v}\|^{2}\right\} d \vec{v} m(d x) \\
= & \left(-\frac{i q}{2 \pi}\right)^{3 n / 2} \int_{\mathbb{R}^{3 n}}\left[\sum_{j=1}^{n}\left\langle\alpha_{j}, w\right\rangle f_{j}\left(\vec{u}+\frac{\langle\vec{\alpha}, y\rangle+\vec{r}}{\sqrt{2}}\right)\right] \\
& \cdot\left[\sum_{k=1}^{n}\left\langle\alpha_{k}, w\right\rangle g_{k}\left(\vec{v}+\frac{\langle\vec{\alpha}, y\rangle-\vec{r}}{\sqrt{2}}\right)\right] \exp \left\{\frac{i q}{2}\left(\|\vec{u}\|^{2}+\|\vec{v}\|^{2}+\|\vec{r}\|^{2}\right)\right\} d \vec{u} d \vec{v} d \vec{r},
\end{aligned}
$$

which, as a function of $y$, is an element of $B(\infty ; m-1)$.

Again, let $m$ be a positive integer, let $F$, and $G$ be elements of $B(2 ; m)$, and let $w \in A$. Then, using (3.9), (3.23), and then (3.9) again, we obtain

$$
\begin{aligned}
&\left(\delta T_{q}(2 ; F)(y \mid \cdot) * \delta T_{q}(2 ; G)(y \mid \cdot)\right)_{q}(w) \\
&=\int_{C_{0}[0, T]}^{a n f_{q}} \delta T_{q}(2 ; F)\left(y \mid \frac{w+x}{\sqrt{2}}\right) \delta T_{q}(2 ; G)\left(y \mid \frac{w-x}{\sqrt{2}}\right) m(d x) \\
&=\int_{C_{0}[0, T]}^{a n f_{q}}\left(-\frac{i q}{2 \pi}\right)^{n / 2} \int_{\mathbb{R}^{n}}\left[\sum_{j=1}^{n}\left\langle\alpha_{j},(w+x) / \sqrt{2}\right\rangle f_{j}(\vec{u}+\langle\vec{\alpha}, y\rangle)\right] \exp \left\{\frac{i q}{2}\|\vec{u}\|^{2}\right\} d \vec{u} \\
& \cdot\left(-\frac{i q}{2 \pi}\right)^{n / 2} \int_{\mathbb{R}^{n}}\left[\sum_{k=1}^{n}\left\langle\alpha_{k},(w-x) / \sqrt{2}\right\rangle g_{k}(\vec{v}+\langle\vec{\alpha}, y\rangle)\right] \exp \left\{\frac{i q}{2}\|\vec{v}\|^{2}\right\} d \vec{v} m(d x) \\
&= \delta T_{q}(2 ; F)(y \mid w / \sqrt{2}) \delta T_{q}(2 ; G)(y \mid w / \sqrt{2}) \\
&-\frac{i}{2 q}\left(-\frac{i q}{2 \pi}\right)^{n} \int_{\mathbb{R}^{2 n}} \sum_{j=1}^{n} f_{j}(\vec{u}+\langle\vec{\alpha}, y\rangle) g_{j}(\vec{v}+\langle\vec{\alpha}, y\rangle) \exp \left\{\frac{i q}{2}\left(\|\vec{u}\|^{2}+\|\vec{v}\|^{2}\right)\right\} d \vec{u} d \vec{v},
\end{aligned}
$$

which, as a function of $y$, is an element of $B(\infty ; m-1)$.

\section{REFERENCES}

[1] M. D. Brue, A Functional Transform of Feynman Integrals Similar to the Fourier Transform, Ph.D. thesis, University of Minnesota, 1972.

[2] R. H. Cameron, The first variation of an indefinite Wiener integral, Proc. Amer. Math. Soc. 2 (1951), 914-924. MR 13,659b. Zbl 044.12103. 
[3] R. H. Cameron and D. A. Storvick, An $L_{2}$ analytic Fourier-Feynman transform, Michigan Math. J. 23 (1976), no. 1, 1-30. MR 53 8371. Zbl 382.42008.

[4] __ Some Banach algebras of analytic Feynman integrable functionals, Analytic functions, Kozubnik 1979 (Proc. Seventh Conf., Kozubnik, 1979) (New York), Lecture Notes in Math., vol. 798, Springer-Verlag, 1980, pp. 18-67. MR 83f:46059. Zbl 439.28007.

[5] _ Feynman integral of variations of functionals, Gaussian random fields (Nagoya, 1990), Ser. Probab. Statist., vol. 1, World Sci. Publishing, 1991, pp. 144-157. MR 93b:28035. Zbl 820.46045.

[6] K. S. Chang, Scale-invariant measurability in Yeh-Wiener space, J. Korean Math. Soc. 19 (1982; 1983), no. 1, 61-67. MR 85k:28020. Zbl 525.28016.

[7] T. Huffman, C. Park, and D. Skoug, Analytic Fourier-Feynman transforms and convolution, Trans. Amer. Math. Soc. 347 (1995), no. 2, 661-673. MR 95d:28017. Zbl 880.28011.

[8] Convolutions and Fourier-Feynman transforms of functionals involving multiple integrals, Michigan Math. J. 43 (1996), no. 2, 247-261. MR 97g:28022. Zbl 864.28007.

[9] _ Convolution and Fourier-Feynman Transforms, Rocky Mountain J. of Math. 27 (1997), no. 3, 827-841. Zbl 980.21808.

[10]___ Generalized transforms and convolutions, Internat. J. Math. Math. Sci. 20 (1997), no. 1, 19-32. MR 97k:46047. Zbl 970.49513.

[11] G. W. Johnson and D. L. Skoug, An $L_{p}$ analytic Fourier-Feynman transform, Michigan Math. J. 26 (1979), no. 1, 103-127. MR 81a:46050. Zbl 409.28007.

[12] - Scale-invariant measurability in Wiener space, Pacific J. Math. 83 (1979), no. 1, 157-176. MR 81b:28016. Zbl 414.60066.

[13] C. Park, D. Skoug, and D. A. Storvick, Relationships Among the First Variation, the Convolution Product, and the Fourier-Feynman Transform, Rocky Mountain J. of Math. 27 (1997), no. 3, 827-841.

[14] J. Yeh, Convolution in Fourier-Wiener transform, Pacific J. Math. 15 (1965), 731-738. MR 32\#1514. Zbl 128.33702.

[15] I. Yoo, Convolution and the Fourier-Wiener transform on abstract Wiener space, Rocky Mountain J. Math. 25 (1995), no. 4, 1577-1587. MR 97j:28032. Zbl 855.28006.

Kim AND Ko: Department of Mathematics, Yonsei University, SeOul 120-749, Korea

PARK: DePARTMENT OF MATHEMATICS AND STATISTICS, MiAMI UNIVERSITY,OXFORD,OH 45056, USA

SKOUG: DePARTMENT OF MATHEMATICS AND STATISTICS, UNIVERSITY OF NEBRASKA, LINCOLN, NE 68588, USA 


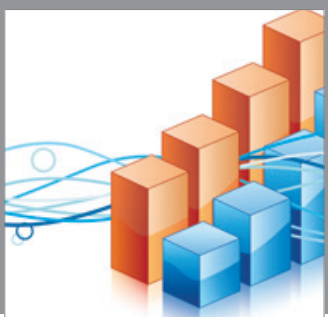

Advances in

Operations Research

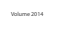

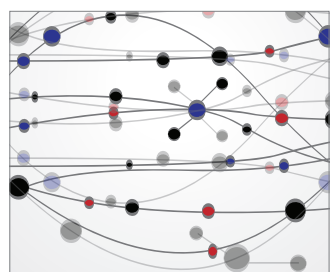

\section{The Scientific} World Journal
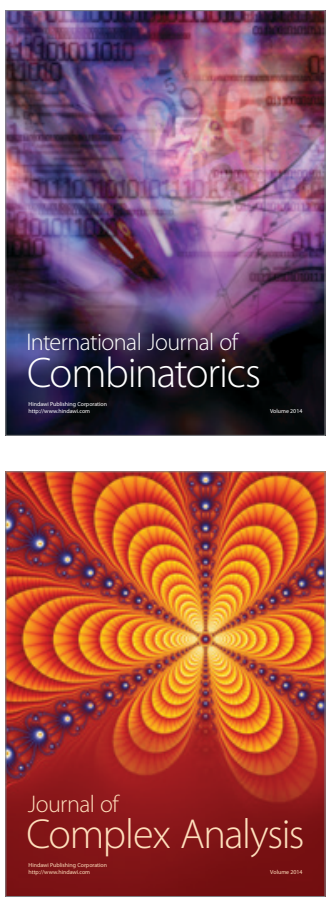

International Journal of

Mathematics and

Mathematical

Sciences
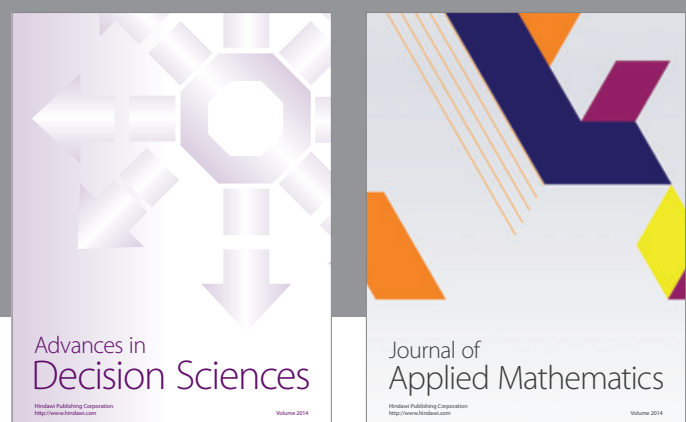

Journal of

Applied Mathematics
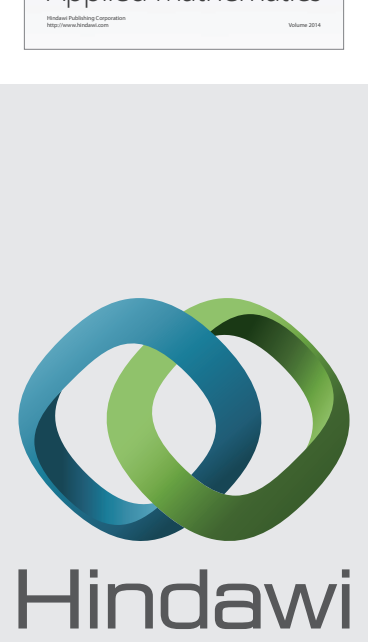

Submit your manuscripts at http://www.hindawi.com
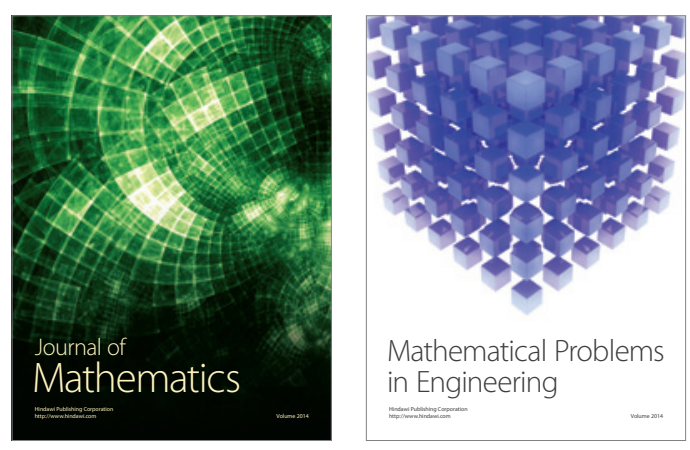

Mathematical Problems in Engineering
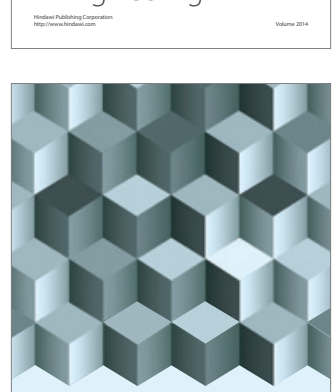

Journal of

Function Spaces
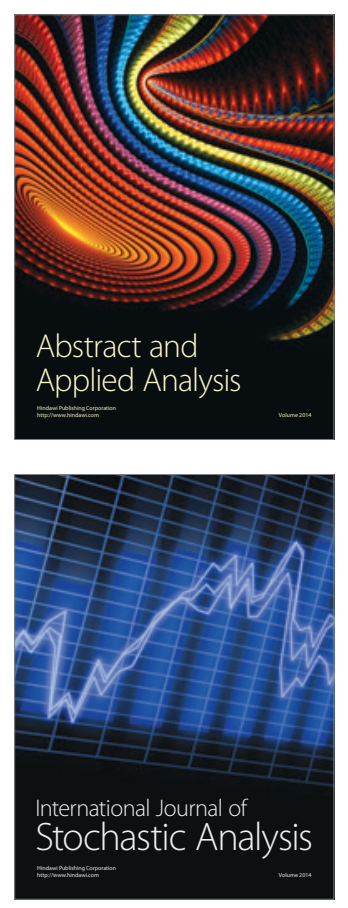

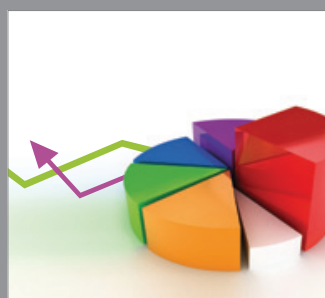

ournal of

Probability and Statistics

Promensencen
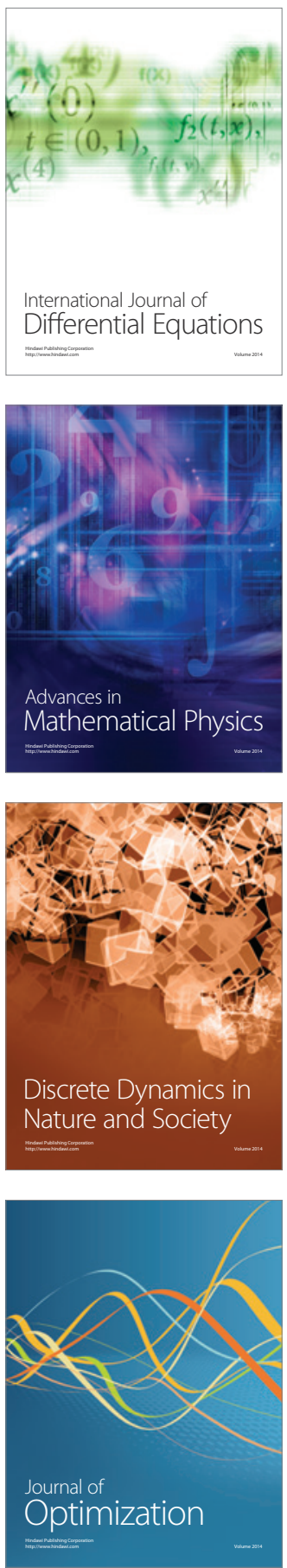